Kauko K. Mäkinen, MS, PhD,,$^{1,2^{*}}$ Kauko P. Isotupa, DDS, ${ }^{2}$ Taina Kivilompolo, $\mathrm{RDH},{ }^{3}$ Pirkko-Liisa Mäkinen, $\mathrm{MS},{ }^{4}$ PhD, Satu Murtomaa, DDS, ${ }^{5}$ Juhani Petäjä, DDS, ${ }^{6}$ Jukka Toivanen, DDS ${ }^{3}$ Eva Söderling, MS, $\mathrm{PhD}^{2}$

${ }^{1}$ University of Michigan, Ann Arbor, MI; ${ }^{2}$ Institute of Dentistry, University of Turku, 20520 Turku, Finland; ${ }^{3}$ Pello County Health Center, Pello, Finland, ${ }^{4}$ Uusikaupunki, Finland ${ }^{5}$ Rovaniemi Rural County Health Center, Rovaniemi Rural County; ${ }^{6}$ Rovaniemi Town Health Center, Rovaniemi Rural County; * corresponding author: kauko.makinen@uusikaupunki.fi

Spec Care Dentist 22(5)187-193, 2002

\section{The effect of polyol-combinant saliva stimulants on $S$. mutans levels in plaque and saliva of patients with mental retardation}

\section{INTRODUCTION}

$T$ he oral health of residents in mental hospitals and psychiatric institutions are the responsibility of the nursing staff in these institutions; however, people who are mentally disabled and are cared for at home by family members may also suffer from poor oral hygiene. Among the most important oral health problems of this cohort are periodontal infections and dental caries, which consume substantial health care resources and cause suffering and pain. The ability to treat and prevent dental caries and to improve oral hygiene among people with severe disabilities are limited. These problems will be aggravated if there is a decline in the effectiveness of the innate chemical defenses of the patient's oral secretions, as often occurs with hyposalivation.

Several studies ${ }^{1-5}$ have shown that substituting sucrose in chewable dietary items with certain dietary polyols (such as xylitol, D-glucitol (sorbitol) and erythritol) can decrease the incidence and symptoms of dental caries. Only two studies ${ }^{6,7}$ have dealt with the effect of such polyols on the status of oral hygiene in subjects who are mentally disabled. One study ${ }^{6}$ suggested that the use of xylitol-containing saliva stimulants may improve oral hygiene, while the other ${ }^{7}$ suggested that xylitol-containing stimulants may be more effective than erythritol-containing ones.

The scientific information on the relative effect of various polyols on the growth of caries-inducing organisms such as mutans streptococci is scant. Apart from the study by Mäkinen et $a l .,{ }^{7}$ no other study has investigated the effect of $S$. mutans on all the polyols used in the present study (erythritol, xylitol and sorbitol) in people who are mentally disabled. The objective of the present study was to investigate the effect of a 64-day program involving saliva stimulants that contained xylitol or sorbitol, or mixtures of xylitol and erythritol or sorbitol and erythritol, on salivary and plaque levels of mutans streptococci in a cohort of subjects who were mentally disabled. The results of this study were expected to increase knowledge on the selective effect of dietary polyols on dental caries. The polyols (polyalcohols) used in the present study-erythritol, xylitol, and sorbitol-represent the four-carbon tetritols, the five-carbon pentitols, and the six-carbon hexitols, respectively, with these terms reflecting the number of hydroxyl groups present in the polyol molecule. According to the proposed scientific tenet, ${ }^{5}$ the effects of xylitol and sorbitol on dental plaque and dental caries can be explained - and are even predictable - based on the pentitol-hexitol theory of polyol effects. The inclusion of a tetritol molecule 
in the present study design was expected to further elucidate polyols' mechanism of action on dental plaque and dental caries.

\section{MATERIALS \& METHODS}

This study was approved by the Human Subjects Committee of the University of Turku Medical School and by the health authorities of the three public, community health centers that participated in this study. Participation in the program was voluntary. No participant was asked to leave the program during its duration. For all of the participants who were mentally retarded, informed consent for participation in the study was obtained from parents, guardians, or the health care personnel in charge. Participants who were not disabled also provided informed consent.

\section{Study site}

This study was a collaborative effort between three public community health centers located in the Lapp territory in Northern Finland. One of the communities, Pello county, is a rural area of about 5,000 inhabitants. The other two were the town of Rovaniemi (population 30,000) and the neighboring rural county of Rovaniemi (population 12,000).

\section{Description of Participants}

Participants in this study were 93 patients of the three health centers, 3 healthy family members who were related to the patients and 2 staff members from the Centers. A total of 98 participants, almost evenly representing the three health centers, were available at bascline. The five healthy participants were recruited to increase compliance among the participants who were disabled. Most participants suffered from varying degrees of mental retardation $(25$ had a severe mental disability). Twenty of the subjects had Down syndrome. Two of the subjects who did not have a mental disability had been diagnosed with Sjögren's syndrome (the Sjögren's syndrome status was a mere chance and had no influence on their inclusion in the study).

The health centers' requirement of recruiting all consenting, mentally disabled participants in this study resulted in some heterogeneity in the participant base. There was no significant difference in the mean ages of the experimental

Table 1. Demographic and physiologic properties of the study population by group at Baseline

\begin{tabular}{ccccc}
\hline Group & $n$ & $\begin{array}{c}\text { Age (years) } \\
\text { (Mean } \pm \text { SD) }\end{array}$ & $\begin{array}{c}\text { DMF score } \\
\text { (Mean } \pm \text { SD) }\end{array}$ & $\begin{array}{c}\text { Whole saliva flow rate }^{c} \\
(\mathrm{ml} / \mathrm{min}) \text { (Mean } \pm \text { SD) }\end{array}$ \\
\hline $\mathrm{X}$ & 26 & $29 \pm 18$ & $12.7 \pm 11.5$ & $0.8 \pm 0.7^{\mathrm{d}}$ \\
$\mathrm{S}$ & 24 & $34 \pm 13$ & $16.2 \pm 9.7$ & $0.5 \pm 0.5^{\mathrm{d}}$ \\
$\mathrm{XE}$ & 22 & $32 \pm 13$ & $12.3 \pm 8.9$ & $0.5 \pm 0.3$ \\
$\mathrm{SE}$ & 23 & $30 \pm 12$ & $11.9 \pm 10.3$ & $0.7 \pm 0.6$ \\
\hline
\end{tabular}

\footnotetext{
a The groups did not differ significantly (One-Way Analysis of Variance, $p=0.530$ ).

b The groups did not differ significantly (One Way Analysis of Variance, $p=0.456$ ).

c The groups did not differ significantly (One Way Analysis of Variance, $p=0.268$ ), The values refer to paraffin-stimulated whole saliva.

d The flow rates of two subjects who had Sjogren's syndrome (one in Group X, the other in Group S) are not included. For these data, $n=25$ for Group $X$ and $n=23$ for Group $\mathrm{S}$.
}

groups (Table 1). Among the participants who were mentally disabled, neither the degree of mental disability nor medication usage differed significantly. These participants received typical psychopharmaceuticals (sedatives) used in most industrialized countries. The five healthy participants were not taking any

The mean decayed, missing or filled (DMF) index scores detcrmined near baseline for the participants did not differ significantly. The mean baseline flow rates of stimulated whole (Table 1).

Questionnaires documented that all participants were similar with regard to oral hygiene habits (tooth brushing), use of dentifrices, and intake of sweet and acidic items. The oral ygiene regimen of the participants during the 64-day erely disabled patients were supervised. The use of for participants before the in this regard. Most patients" daily chores were superviscd. (daily chores: oral hygiene, eating, dressing, etc.) The patients received oral health care at the centers' nursing homes or at homes of the participating families. The participants' dental records were available and kept at the health centers.

Each of the four study groups had approximately the same Saliva stimulants

The saliva stimulants used in this study were chewable, circular, relatively fragile tablets (weight $=1$. I grams $[\mathrm{g}]$ ) that designed to easily fragment in the mouth, allowing the (erythritol, xylitol, or sorbitol) to dissolve quickly in wincated that the tablets fragmented and dissolved in saliva participants who had Sjögren's syndrome). The easily fragmented tablets were used to achieve a difference between the chewable stimulants and the swallowed pills taken regularly most of the patients. This was done to ensure a physical breakdown of the saliva stimulants in an environment separate from other pills. The patients were used to swallowing rounded, hard and polished pills (medicines). The present tablets were made larger, softer, and brittle (friable) so that they fragmented and dissolved quickly in saliva. The swallowing of tablets would have made local oral effects of tablets impossible.

The tablets contained 49\% polyol (single polyol or a 50:50 mixture of two polyols; see below), 49\% polydextrose (Purac Biochem's STA-LITE ${ }^{12}$ III-F; Gorinchem, The Netherlands) as a bulking agent, and small amounts of binding agents and peppermint aroma. The polydextrose was reported to contain a maximum of $6 \%$ of sorbitol and D-glucose and a minimum of $90 \%$ polydextrose (on dry substance; average degree of polymerization maximum 9-10).

Fennobon Oy (Karkkilá, Finland) made the polyol tablets that were used in this study. Four types of tablets were made; they contained 
xylitol (X), sorbitol (S), a mixture of xylitol and erythritol (XE), or a mixture of sorbitol and erythritol (SE). The xylitol was obtained from Xyrofin Oy (Kotka, Finland), sorbitol from Merck (Darmstadt, Germany), and erythritol was obtained from Cerestar Italia (Ferrara, Italy). The purity of the polyols, the polydextrose, and other matcrials used in the manufacturing of the tablets conformed to the criteria on food additives according to the European Commission directives, or complied to USP/NF and $\mathrm{FCC}$ monographs for the named substances. On completion, the X-and S-tablets contained $49 \%$ of xylitol or sorbitol, respectively; the XE-tablets contained about $24.5 \%$ xylitol and ca. $24.5 \%$ crythritol; and the SE-tablets contained ca. $24.5 \%$ of sorbitol and ca. $24.5 \%$ erythritol. A Finnish government-administered agency (VTT, Helsinki) independently conducted liquid chromatographic analyses on random tablet samples to verify that the samples contained the intended quantities of polyols.

The tablets assigned to be used by each participant during the study were packed in a total of 64 number-coded plastic bags, with each bag containing one participant's daily dose of 10 tablets.

\section{Study design}

After the participants or their guardians signed the informed consent documents, each participant was systematically assigned to one of four test groups with the exception that participants who lived in the same household were assigned to the same group. The participants who were mentally disabled regularly visited the participating dental health center for chcckups and treatment. Participants who were not disabled regularly visited these or private clinics. The attending health care personncl were well-acquainted with the participants' behaviors and oral health status.

\section{Distribution of the saliva stimulants and instruction}

At each health center, the oral hygienist distributed the saliva stimulants at baseline, either to the participants directly or to the guardian, and provided instruction about the use of the products. The dosage of the tablets was made progressive: on the first seven days of the study the participants were instructed to use one tablet five times a day; beginning on Day 8 and continuing through Day 64, the participants were told to chew two tablets five times a day. The daily consumption level of xylitol and sorbitol in the $X$-and $S$-groups for each subject was 5.4 grams during the final 57 days of the study. The daily consumption levels per subject in the XE- and SE-groups was $2.7 \mathrm{~g}$ /polyol (the total polyol consumption level was thus 5.4 $\mathrm{g} /$ day/ subject) during the last 57 days of the study.

To prolong the clearance of the polyols from the moutl, participants were instructed to take the tablets separately but consecutively (compliance could not be verified in all cases). In general, the tablets were taken following main meals and snacks of the participants; accordingly, the chewing times were near 8:00 a.m., 12:00 p.m., 3:00 p.m., 6:00 p.m., and 9:00 p.m. The last chewing episode took place in the evening after oral hygiene care. The subjects were advised, whenever possible, to suck and chew the tablets slowly.

Participants who were mentally disabled received the tablets from parents, guardians, or resident attendants, who were asked to supervise the use of the product. Other participants used the tablets without supervision but were instructed with regard to their use. The participants were asked to make no other changes in their normal dietary regimen or in their previous oral hygiene habits. They were asked not to use any additional oral hygiene procedures and were advised to use no other commercial products containing erythritol, xylitol, or sorbitol during the study (participants also were asked to refrain from using these products for one month before the study).

The participants or their guardians also were given a simple diary in which they were asked to enter daily information about any side effects, use of sweet and acidic food items, oral hygiene, and the exact time the saliva stimulant was chewed. The oral hygienist maintained regular contact with the subjects or their families via phone calls, visits, and postcards.

Before coming for the baseline examination, the participants were asked not to brush their teeth for 36 hours. The participants visited the health center's dental clinic on Days 36 and 64 when the study's intermediate and endpoint examinations were carried out. The no brushing schedule was also used for the intermediate and endpoint examinations. On all test days, the examinations and tests were conducted between 8:00 a.m. and 3:00 p.m.

\section{Collection, analysis of plaque samples}

Each appointment began with a collection of 1 - to 2milligrams $(\mathrm{mg})$ of plaque with sterile toothpicks from four separate tooth sites, interproximal regions and cervical margins, from the premolars and representing all four quadrants. The sample-containing tip of the four toothpicks from each quandrant was cut off with sterile scissors and pooled into a transport medium $(0.5$ milliliters [ $\mathrm{ml}])$ for the cultivation of streptococci. The transport vials were immediately frozen in solid carbon dioxide (ca. $-79^{\circ} \mathrm{C}$ ) and were stored below $-20^{\circ} \mathrm{C}$ until thawed for analyses within one month. The transport medium contained tryptic soy broth (Difco, Detroit, Mich.) with 10\% glycerol (volume per volume).

For the microbiologic analyses of the plaque collected with tooth picks, the contents of the transfer tubes were thawed and vortexed thoroughly for $1 \mathrm{~min}$. To detach the plaque from the toothpicks and to disrupt bacterial aggregates, the samples were additionally given a 10 -second ultrasonic treatment at $+4{ }^{\circ} \mathrm{C}$. An earlier study ${ }^{8}$ indicates that the levels of mutans streptococci are not affected by the laboratory methods used in this study. Following serial, ten-fold dilutions, the bacteria were plated on mitis salivarius agar (Difco) containing bacitracin (MSB agar). ${ }^{9}$ The plates were incubated for two days in a $7 \%$ carbon dioxide atmosphere at $37^{\circ} \mathrm{C}$.

The bacteria were identified based on colony morphology and counted using a stereomicroscope. The identification of $S$. mulans and S. sobrimus was performed as described earlier by Fujiwara et al. ${ }^{10}$ Classification of $S$. mutans was based on consistent findings of "rough" colony morphology on the MSB plate, on positjve fermentation in the presence of $D$-glucitol, $D$ mannitol, raffinose, and melibiose, and on negative dextran agglutination. The identification of $S$. sobrinus was based on its "smooth" colony morphology on the MSB plate, on its positive fermentation of $D$-mannitol but negative fermentation of raffinose and melibiose, and on positive dextran agglutination. The detection limit of the assay was 20 colony-forming units 
(cfu) per plaque sample. These tests included counting of the total number of plaque streptococcal colonies as a background variable. In this study, the plaque counts of $S$. mutans also were documented as a percentage of the total streptococcal colonies.

\section{Collection, analysis of saliva samples}

Participants provided a whole saliva sample using 2-minuteparaffin stimulation. The participants were instructed to chew a 1.2-gram piece of paraffin continuously as if it were chewing gum. The experimental groups did not differ regarding the performance of saliva stimulation. The volume of saliva was recorded. The level of salivary mutans streptococci was tested by means of the Orion Diagnostica Dentocult $\mathrm{SM}^{(\overline{\mathrm{Q}}}$ strip mutans test (Espoo, Finland). The number of mutans streptococci, expressed per milliliter of saliva, was evaluated independently by two experienced raters according to the test kit instructions provided by the manufacturer. The consensus of the raters was taken as the final result (the raters independently scored the test strips with a $96 \%$ agreement).

\section{Blinding}

The participants (and their guardians, parents, and attending health care personnel) were told that the purpose of the study was to compare erythritol-, xylitol-, and sorbitol-containing tablets, but were not told to which polyol group the participants were assigned. The clinical and laboratory personnel involved in analytical and sampling procedures likewise were not aware of the product assignment. The results of the baseline determinations were not available for examiners at subsequent determinations.

\section{Statistical procedures}

The significance levels between means (determined at Baseline, Day 36 and Day 64) were studied using the Jandel SigmaStat 2.0 program (1992-1995), which analyzes the data based on its normality and other characteristics. The procedures included the application of the Friedman Repeated Measures Analysis of Variance on Ranks (for salivary counts of mutans streptococci), and the One-Way Repeated Measures Analysis of Variance (for plaque counts of microorganisms). In addition, a Repeated Anova Test was performed to determine differences over time between the X-group and the XE-group. The significance levels between mean values at Baseline (between

Table 2. Effect of polyol-containing saliva stimulants on S. mutans in interproximal plaque.

\begin{tabular}{cccccc}
\hline Group & $\mathrm{n}$ & Baseline $^{\mathrm{a}}$ & Day 36 & Day 64 & -value $^{\text {b }}$ \\
$\mathrm{X}$ & 26 & $3.08 \pm 2.79$ & $2.37 \pm 2.72$ & $2.00 \pm 2.71$ & 0.001 \\
$\mathrm{~S}$ & 24 & $4.10 \pm 2.42$ & $4.74 \pm 2.23$ & $4.35 \pm 2.46$ & 0.205 \\
$\mathrm{XE}$ & 21 & $3.77 \pm 2.00$ & $2.47 \pm 2.34$ & $2.51 \pm 2.41$ & 0.004 \\
$\mathrm{SE}$ & 23 & $3.16 \pm 2.66$ & $3.14 \pm 2.55$ & $2.44 \pm 2.60$ & $0.092^{\mathrm{c}}$ \\
\hline
\end{tabular}

a The values shown are means $\pm S D$ of $\log _{10}$ counts; the number $(n)$ of participants is indicated. The baseline values did not differ significantly (Kruskal-Wallis One Way Analysis of Variance; $p=0.431$ ).

b The $p$-values, determined by means of One-Way Repeated Measures Analysis of Variance, show the statistical differences between baseline and treatment period in each experimental group.

$c$ The difference between baseline and the treatment period may be considered indicative. experimental groups) were examined by means of the KruskalWallis One-Way Analysis of Variance. The confidence limit of $95 \%$ was taken as a significant difference $(p<0.05)$. Additionally, a paired $t$-test was used to measure the ificance of mean values between Baseline and Day 36, 6y 36 and Day 64, and between Baseline and Day participant as his/her own control. This was regarded as an propriate procedure since individual differences in oral biologic parameters were considerable in the studied patient base.

A $t$-test was used to compare means of experimental groups at different time points.

\section{RESULTS}

Five participants out of the 98 originally examined at Baseline were not present for the Day-64 examinations. One of these participants belonged to the X-group, three participants to the $\mathrm{XE}$-group, and one participant to the SE-group. In each case, the reason for dropping out was noncompliance, as determined the attending personnel. None of the remaining participants stomach problems; no such problems were reported to the health centers by the guardians of the patients either. The compliance of the participants with the therapeutic regimen as regarded as good in about $90 \%$ of the population. This was fo the supervision provided by parents, guardians, considered satisfactory in about $10 \%$ of the patients who completed the program. In general, the authors suspect that pliance was improved because the participants were accustomed to taking daily medication. This compliance level was considered to be higher than that in similar studies ${ }^{6,7}$ among this type of population.

\section{Plaque counts of $\boldsymbol{S}$. mutans} of $S$. mutans in interproximal plaque is shown in Table 2. The ence of these bacteria in plaque decreased significantly in ants receiving $\mathrm{X}$-tablets $(p=0.001)$ and those receiving Xe-tablets $(p=0.004)$, while no such reduction was observed in participants who had received S- or SE- tablets. The bacterial counts had already decreased significantly $(p<0.05)$ in the Xand the XE-groups by Day 36 . No such effect was observed in the other test groups.

The Figure shows bacterial counts obtained from interproximal plaque samples collected at Baseline (1), Day 36 (2), and at Day 64 (3). The mean values did not differ significantly at baseline (One-Way Analysis of Variance; $p=0.817$ ). One-Way Repeated Measures Analysis showed the following: S-group; the differences between mean values were greater than would be expected by chance, i.e. there was a statistically significant difference $(p=0.004)$. $\mathrm{X}-$, XE-, and SE-groups; the differences in the mean values between baseline and subsequent tests were not great enough to exclude the possibility that the differences resulted from random sampling variability (the $p$-values for these three groups were $0.393,0.252$, and 0.218 , 

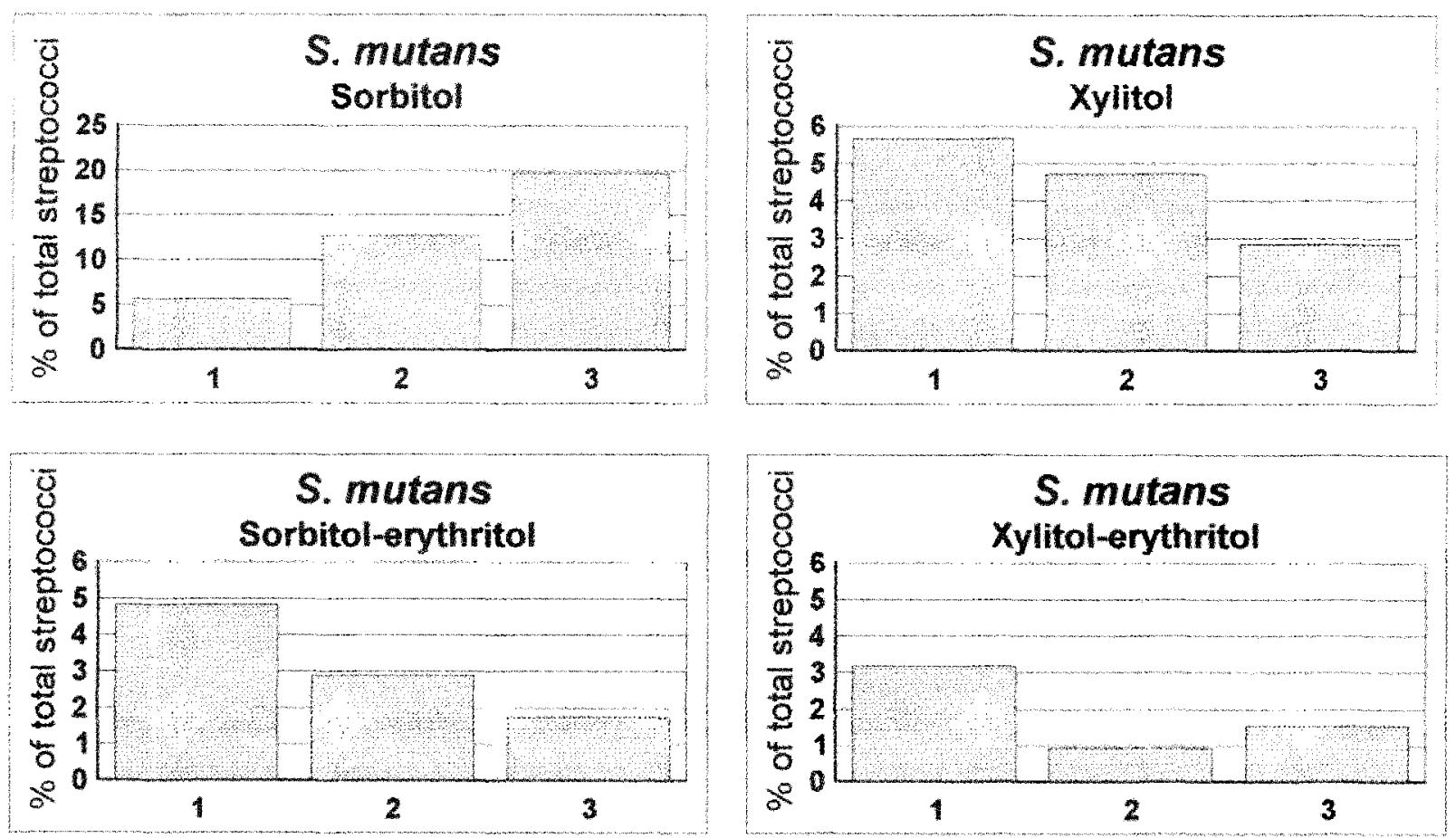

Figure. Effect of two-month usage of polyol-containing saliva stimulants on the relative occurrence of $S$. mutans in interproximal dental plaque (in total streptococcal counts percentage).

respectively). However, the groups differed significantly at Day 64 (see text). The standard deviation values for Baseline, Day 36, and Day 64, respectively, were as follows: S-Group: $\pm 7.4, \pm 18.6, \pm 29.7$. X-Group: $\pm 13.5, \pm 11.1, \pm 6.5$. SE-Group: $\pm 9.9, \pm 6.2, \pm 3.0$. XE-Group: $\pm 6.2, \pm 2.4, \pm 4.6$.

The analysis of total plaque streptococci showed that these organisms were abundant in all participants' toothpick samples. Accordingly, it was considered justified to express the counts of $S$. mutans against this material control. The Figure shows that the percent share of $S$. mutans increased significantly in the S-group over time, while in other test groups the percent share of these organisms decreased. Owing to the large standard deviation, these decreases were not statistically significant. The relative portion of $S$. mutans of total streptococci at endpoint was significantly higher in the Sgroup compared with the X-group $(p=0.007)$,

with the XE-group $(p=0.010)$, and with the SE-group $(p=0.007)$. The endpoint percentages did not differ significantly between $X$ and XE $(p=0.475)$, and between $X$ or SE $(p=0.457)$. Consequently, the presence of xylitol and xylitol:erythritol in the tablets was associated with a reduction in the relative occurrence of $S$. mutans in interproximal dental plaque compared with the use of either sorbitolcontaining stimulants.

\section{Plaque counts of Streptococcus sobrinus}

The effect of the usage of the polyolcontaining saliva stimulants on the levels of Streptococcus sobrinus in interproximal plaque is shown in Table 3. The S. sobrinus species is normally present in dental plaque in smaller numbers than $S$. mutans. Consequently, the numbers of the cells of $S$. sobrinus in dental plaque also were fewer in this study. Use of the saliva stimulants had no significant effect on these bacterial counts. However, the use of S-tablets was associated with somewhat elevated counts, which over time approached significance $(p=$ $0.053)$.

\section{Salivary counts of mutans streptococci}

The paraffin-stimulated whole saliva of the participants was tested for mutans streptococci using the Orion Diagnostica Dentocult $\mathrm{SM}^{\circledR}$ Strip Mutans test. We chose to use this test to investigate the suitability of the strip mutans procedure in the

Table 3. Effect of polyol-containing saliva stimulants on $S$. sobrinus in interproximal plaque.

\begin{tabular}{cccccc}
\hline Group & $n$ & Baseline $^{a}$ & Day 36 & Day 64 & $p$-value \\
$X$ & 26 & $1.11 \pm 2.33$ & $1.07 \pm 2.26$ & $1.25 \pm 2.62$ & 0.757 \\
$S$ & 24 & $1.41 \pm 2.55$ & $1.48 \pm 2.65$ & $1.82 \pm 2.73$ & $0.053^{\mathrm{c}}$ \\
$\mathrm{XE}$ & 21 & $1.71 \pm 2.61$ & $1.71 \pm 2.64$ & $1.67 \pm 2.66$ & 0.830 \\
$\mathrm{SE}$ & 23 & $1.28 \pm 2.60$ & $1.18 \pm 2.58$ & $1.27 \pm 2.47$ & 0.186 \\
\hline
\end{tabular}

a The values shown are means $\pm S D$ of $\log _{10}$ counts; the number $(n)$ of participants is indicated. The baseline values did not differ significantly between groups (Kruskal-Wallis One Way Analysis of Variance; $p=0.875$ ).

$b$ The $p$-values, determined by means of One-Way Repeated Measures Analysis of Variance, show the statistical differences between baseline and treatment period in each experimental group.

c The difference from baseline approaches significance. 
Table 4. Effect of polyol-containing saliva stimulants on $S$. mutans in stimulated saliva as determined by means of a commercial test strip (Dentocult SM).

\begin{tabular}{cccccc}
\hline Group & $\mathrm{n}$ & Baseline $^{\mathrm{a}}$ & Day 36 & Day 64 & $p$-value \\
$\mathrm{X}$ & 26 & $1.96 \pm 1.11$ & $1.56 \pm 1.33$ & $1.50 \pm 1.30$ & 0.010 \\
$\mathrm{~S}$ & 24 & $2.12 \pm 1.15$ & $2.35 \pm 0.98$ & $2.00 \pm 1.32$ & 0.430 \\
$\mathrm{XE}$ & 22 & $2.52 \pm 0.93$ & $2.00 \pm 1.00$ & $1.85 \pm 1.18$ & 0.017 \\
$\mathrm{SE}$ & 23 & $2.09 \pm 1.24$ & $1.86 \pm 1.21$ & $1.91 \pm 1.23$ & 0.430 \\
\hline
\end{tabular}

a The values shown are means $\pm S D$ of bacterial scores $(0,1,2$, or 3$)$ determined as described in the test kit manual; the number ( $n$ ) of participants in each experimental group is indicated. The baseline values did not differ significantly (Kruskal-Wallis One Way Analysis of Variance on Ranks, $p=0.337$ ). The tested whole saliva was obtained by paraffin stimulation.

b The $p$-values, determined by means of Friedman Repeated Measures Analysis of Variance on Ranks, show the statistical differences between baseline and treatment period in each experimental group.

monitoring of saliva levels of those bacteria in this type of study.

Levels of mutans streptococci in stimulated saliva did not differ significantly between the experimental groups at Baseline (Kruskal-Wallis one-way analysis of variance on ranks; $p=0.337$ ). The saliva test showed a significant difference between the mean scores measured before and after treatment with $\mathrm{X}$ - and XE-tablets, while no such difference was observed after use of the S- or SE-tablets (Table 4). At Day 64 , the difference between Groups $X$ and XE on one hand, and that recorded between Groups $S$ and SE on the other, was not significant. The consumption of the X- and XEtablets was associated with a significant $(p<0.05)$ reduction in the bacterial counts already during the first 36 days of the study. This was not observed in other test groups. Accordingly, the use of xylitol-containing saliva stimulants was associated with a significant reduction in the salivary scores of mutans streptococci compared with the use of stimulants that contained sorbitol; the use of the latter resulted in no significant change in bacterial scores.

\section{DISCUSSION}

This study showed that 64-day use of saliva stimulants containing xylitol (a polyol, or sugar alcohol, of the pentitol type) was associated with a significant reduction in the saliva and plaque levels of mutans streptococci in a cohort of subjects who were mentally disabled. Saliva stimulants that contained sorbitol (a sugar alcohol of the hexitol type), either as the predominant bulk sweetener ( $\mathrm{S}$-tablets), or in a $1: 1$ mixture with erythritol (SE-tablets), had no significant effect on the measured bacterial counts. Sorbitol behaved in this study as an inert bulk sweetener with no special effect on mutans streptococci. The results in this study should be evaluated understanding that all tested saliva stimulants contain $49 \%$ polydextrose as a bulking agent, which in turn contained about $6 \%$ sorbitol and D-glucose. The presence of these monosaccharides in the tested stimulants did not inhibit the "xylitol effect."

The present study constitutes the first clinical investigation that attempted to determine the effect on mutans streptococci of saliva stimulants containing tetritol-, pentitol-, and hexitoltype sugar alcohols. Previous studies ${ }^{1-5}$ have shown that the five-carbon sugar alcohol xylitol is more effective than the six-carbon sorbitol in reducing both the number of mutans streptococci in saliva and in the plaque.

Information on the oral biologic effects of the four-carbon homologue, erythritol, is scant. The published reports suggest, however, that erythritol may be regarded as harmless from the cariogenic point of view. ${ }^{7.1}$ Unpublished results from the authors" laboratory suggest that erythritol may actually exert an inhibitory effect on mutans streptococei that is not based on the same biochemical mechanism suggested for xylitol-associated inhibition.

The present study confirms carlier findings on the differences between xylitol and sorbitol in their effect on mutans streptococci. Previous studies $^{4}$ and our study suggest that xylitol and mutans streptococci have a relationship that has not been observed between these bacteria and six-carbon sugar alcohols. This suggestion is substantiated by the absence of any strong and consistent inhibitory effect of xylitol on $S$. sobrinus and total streptococci counts. The relationship between xylitol and S. sobrinus, however, should be studied using larger cohorts than those used in this study.

The present results also showed that the addition of erythritol to a xylitol product (XE-tablets) did not weaken the ability of xylitol to reduce the growth of mutans streptococci. On the other hand, the presence of erythritol in the SE-tablets allowed a potential, specific erythritol effect on mutans streptococci (Figure). Consequently, it appears that the fivecarbon pentitol nature of the xylitol molecule constitutes a specific chemical determinant that forms the basis of the "xylitol effect," and that the four-carbon erythritol molecule also may specifically inhibit $S$. mutans.

When comparing the effects of xylitol and sorbitol on mutans streptococci, one should be aware of the "non-glucose" nature of xylitol. Sorbitol, with its "glucose-polyol" nature does not exert similar inhibitory effects on mutans streptococci as xylitol does. ${ }^{5}$ The results of this study suggest that the Dentocult SM ${ }^{\text {S }}$ Strip Mutans test can be used to cvaluate the effect of polyol-type sugar substitutes on caries-associated streptococci.

This study and an earlier one ${ }^{7}$ carried out in the same district in Northern Finland also involving participants who were mentally disabled showed that habitual use of the saliva stimulants containing xylitol may significantly improve the efficacy of the existing caries-preventive strategies used with patients who are mentally disabled. Based on the reports of several patients and their parents or guardians, the saliva stimulants used in these studies were considered an approach that improved dental health. Further research involving people who are mentally disabled is needed, for example, to explain the relatively frequent detection in this study of measurable counts of $S$. sobrinus in the plaque. It appears that high counts of these bacteria were observed in patients with poor oral hygiene. The participants who were mentally disabled generally showed higher bacterial counts than those reported among people with no disabilities. ${ }^{12}$ The relationship between S. mutans and erythritol also requires further study. 


\section{CONCLUSIONS}

The present study indicates that xylitol-containing saliva stimulants were more effective in reducing the saliva and plaque counts of mutans streptococci than were sorbitolcontaining stimulants. Also, the addition of erythritol to a xylitol-containing saliva stimulant did not prevent the manifestation of the inhibitory effect of xylitol on bacterial counts. It is possible that erythritol exerts a specific inhibitory effect on mutans streptococci. This effect may chemically differ from that exhibited by xylitol, because tetritol-and pentitol-type polyols may be expected to behave differently in a microbial system. However this possibility must be substantiated in other studies.

The results of this study also support the tenet that a special relationship exists between the five-carbon xylitol molecule and the metabolism of mutans streptococci.

Patients who are mentally disabled challenge their caretakers to maintain their oral health. There are difficulties encountered in instructing these patients with regard to oral health habits so that any favorable changes in oral health should be considered encouraging.

\section{ACKNOWLEDGEMENTS}

This study received financial support from the Finnish government-administered Technology Development Center of Finland.

The informed consent of all participants in this investigation was obtained after the nature of the procedures and possible discomforts and risks had been fully explained, in accordance with the requirements of the Human Subjects Committee of the University of Turku Medical School. Permission for this study also was received from the Board of Health of each of the participating municipalities.

The authors thank Mrs. Maritta Enbuska, Mrs. Riitta Isotupa, Mrs. Sylvi Niemi, Mrs. Päivi Meriläinen-Visuri, Ms Oona Kalo (Medical Laboratory Technologist), and Mrs. Annukka Wallenius for technical assistance. The technical officers (Mr. Paavo Kuusela and Mrs. Eira Laurila) of Fennobon Oy are offered special thanks.

\section{REFERENCES}

1. Mäkinen KK. Prevention of dental caries by xylitol - issues related to health claims. In: Tillotson JE (ed.). America's foods health messages and claims. Boca Raton, Florida: CRC Press, pp 167-92, 1993

2. Birkhed D. Cariologic aspects of xylitol and its use in chewing gum: a review. Acta Odontol Scand 52:116-27, 1994.

3. Tanzer J. Xylitol chewing gum and dental caries. Int Dent $J 45$ (1 Suppl 1):65-76, 1995.

4. Trahan L. Xylitol: a review of its action on mutans streptococci and dental caries - Its clinical significance. Int Dent $J 45$ (Suppl 1):77-92, 1995.

5. Mäkinen KK. Can the pentitol-hexitol theory explain the clinical observations made with xylitol? Med Hypotheses 54:603-13, 2000.

6. Pakkala U, Liesmaa H, Mäkinen KK. The use of xylitol in the control of oral hygiene in mentally retarded children: a clinical and biochemical study. Proc Finn Dent Soc 77:271-7, 1981.

7. Mäkinen KK, Isotupa KP, Kivilompolo T, et al. Comparison of erythritol and xylitol saliva stimulants in the control of dental plaque and mutans streptococci. Caries Res 35:129-35, 2001.

8. Trahan L, Söderling E, Dréan MF, Chevrier MC, Isokangas P. Effect of xylitol consumption on the plaque-saliva distribution of mutans streptococei and the occurrence and long-term survival of xylitol-resistant strains. J Dent Res 71:1785-91, 1992

9. Gold OG, Jordan HV, van Houte J. A selective medium for Streptococcus mutans. Arch Oral Biol 18:1357-64, 1973.

10. Fujiwara T, Sasada E, Mima N, Ooshima T. Caries prevalence and salivary mutans streptococci in 0 -2-year old children in Japan. Community Dent Oral Epidemiol 19:151-4, 1991.

11. Kawanabe J, Hirasawa M, Takeuchi T, Oda T, Ikeda T. Noncariogenicity of erythritol as a substrate. Caries Res 26:35862, 1992

12. Loesche WJ. Dental caries: a treatable infection. Springfield, Illinois: Charles C. Thomas, 1982. 